\title{
Non-Integrability of the 4-Vortex System: Analytical Proof
}

\author{
Jair Koiller ${ }^{1, \star}$ and Sonia P. Carvalho ${ }^{2}$ \\ ${ }^{1}$ Laboratorio National de Computação Cientifica, Caixa Postal 56018, 22290 Rio de Janeiro, RJ, \\ Brazil \\ ${ }^{1}$ Instituto de Matematica da UFRJ, Caixa Postal 68530, 21944 Rio de Janeiro, RJ, Brazil \\ ${ }^{2}$ Departamento de Matematica da UFMG, ICEX, Pampulha, Belo Horizonte, MG, Brazil, \\ 30000
}

\begin{abstract}
An analytical proof is given that the motion of $n$ point vortices in the plane is non-integrable for $n>3$. The basic geometric configuration, which models a situation often found experimentally, consists of two opposite strong vortices and two advected weak vortices. We use "Melnikov's method," as presented by Holmes and Marsden [Commun. Math. Phys. 82, 523-544 (1982)]. The Melnikov integral is explicitly evaluated, by residues, in the limiting situation where one of the weak vortices is very close to one of the primaries.
\end{abstract}

\section{Introduction}

Our aim here is to present a completely analytical proof for the presence of horseshoes in the dynamics of 4 point vortices, using the Melnikov method as presented by Holmes and Marsden [HM]. The hydrodynamical background can be found in Aref [A1-A3]; for the mathematical relevance of this and other nonintegrability problems, see Kozlov [Kz, Kz1]. The Dynamical Systems prerequisite here is the statement (with caveats, see Sect. 4): "the presence of transversal homoclinic points implies nonexistence of further (analytical) integrals of motion." Non-expert readers may find all the relevant material in Guckenheimer and Holmes [GH].

Our choice for a special geometrical setting consists of two strong, opposite vortices, and two weak, advected ones. Two-dimensional flows whose structure is dominated by strong, opposite couples are fairly common: Couder and Basdevant [CB] call those couples "Bachelor couples" in a remarkable double pun (they are very stable and a homage to Prof. Batchelor).

Ziglin [Z1, 1980], using another special configuration, gave a semi-analytical proof for the non-integrability: the Melnikov integral is evaluated numerically.

^ Visitor at the Department of Mathematics, Yale University, 1986-1987, under a CAPES/Brazil fellowship 
Commenting on [HM], Ziglin [Z2, 1987] pointed out that his approach does not posses the "exponentially small difficulty," as these authors have suspected. With due apologies to Prof. Ziglin (since we have followed [HM] on a first technical report [KC1, 1983], we would like nevertheless to present the following excerpt from an extended technical report [KC2, 1985]:

\begin{abstract}
"As far as we know, Ziglin (1980) was the first author to show the nonintegrability of a four vortex problem. Ziglin considers the advection of a particle by the flow generated by three equal vortices. Some objections were raised initially to his approach (Holmes and Marsden, 1982), but it is entirely correct (Marsden, 1984, personal communication). In order to compare our method to his, we make a brief review of his work. Ziglin's perturbation parameter $\varepsilon$ is of geometric character, namely the deviation from the primaries equilateral configuration. When $\varepsilon=0$, the phase portrait of the marker possesses several separatrices, on a frame such that the primaries are at rest. The Melnikov integral was shown to be not equal to zero by a numerical calculation. Subsequently, in a small note (addendum to Khanin, 1982), Ziglin gives a short mathematical argument, to show that the non-integrability of the unrestricted system follows immediately from the persistence of hyperbolic structures."
\end{abstract}

This paper is organized as follows: in Sect. 2 we prove transversal separatrix splitting for a restricted problem. It is intended as a warm-up for non-experts, although we assume that the reader is familiar with [HM]. In Sect. 3 we prove transversal separatrix splitting in the general case; the analysis is much more laborious, but it turns out that the physical interpretation of the result is the same in both cases. In Sect. 4 we discuss the usefulness of Melnikov's method for Hamiltonian systems in the light of Lagrangian intersection theory [W]. The computational details are left to the appendices.

Remark. During the editorial process, a paper by Ottino et al. [O] came to our attention, which could give some perspective to our work. See also, in the same Nature issue, the "News and Views" article by S. Wiggins, whose Fig. 1 is precisely the basic setting of our paper.

\title{
2. Bachelor Couple with a Restricted Problem (and Chaotic Behavior)
}

A single vortex at the origin in the complex plane generates the flow whose trajectories are the solutions of $\dot{z}=i k z /|z|^{2}$. We call $|k|$ the strength of the vortex (in the customary fluid dynamical terminology, the strength or circulation is $2 \pi|k|)$. Following Helmholtz, in order to set up the dynamics of vortex systems, one simply drops out the influence from each vortex on itself.

For instance, two vortices of equal strength but opposite signs in an otherwise irrotational flow move uniformly on parallel lines perpendicular to the line joining their centers (this is an elementary but nonetheless important fact). We fix units so that $k_{1}=-k_{2}=1 / 2,\left|z_{1}-z_{2}\right|=2$, so that the velocity is $1 / 4$. We consider the motion of a marker (i.e., a point of the fluid with zero vorticity) in the flow determined by the primaries $z_{1}, z_{2}$. The following is elementary.

Proposition 1. In a moving coordinate system in which the primaries are at rest at $z_{1}=-z_{2}=i$, the advected particle will obey the Hamiltonian

$$
\begin{aligned}
& \dot{u}=F_{v}, \quad \dot{v}=-F_{u}, \quad F(u, v)=v / 4+(1 / 2) \log r_{1} / r_{2}, \\
& \left(z=u+i v, r_{1}=|z-i|, r_{2}=|z+i|\right) .
\end{aligned}
$$




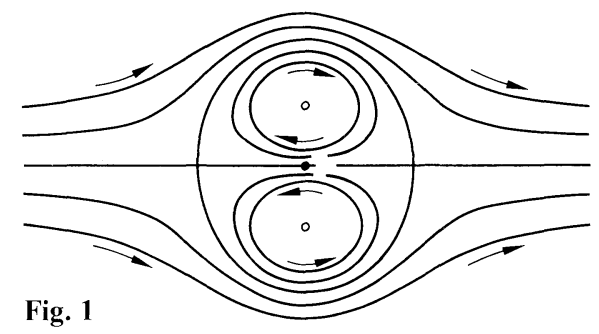

The phase portrait is depicted in Fig. 1. Figure 3 of $[\mathrm{CB}]$ strikingly depicts the qualitative ubiquity of these structures.

Definition. The double restricted problem of four vortices consists of a Bachelor couple $z_{1}, z_{2}$ with two "offsprings" (i.e., two weak vortices) $z_{3}, z_{4}$ of equal strength $\varepsilon \sim 0$ such that their action on the primaries is neglected.

This terminology is borrowed from Celestial Mechanics [WS]. If $\varepsilon$ is set equal to zero, then the equations for the offsprings uncouple, yielding two independent (albeit exactly equal) phase portraits as in Fig. 1. The interaction Hamiltonian for the weak vortices, in the moving frame, is

$$
H^{\varepsilon}\left(p_{1}, q_{1}, p_{2}, q_{2}\right)=F\left(p_{1}, q_{1}\right)+G\left(p_{2}, q_{2}\right)+\varepsilon H^{1}\left(p_{1}, q_{1}, p_{2}, q_{2}\right),
$$

where $z_{3}=p_{1}+i q_{1}, z_{4}=p_{2}+i q_{2}, H^{1}=\log \left|z_{3}-z_{4}\right|, F=G$ as in (1).

We stress again that the perturbation on the primaries has been neglected, but not their mutual action. The point is that Hamiltonian (2) is precisely in the context for homoclinic behavior in interacting " $F-G$ systems" [HM]. We let $\varepsilon=0$, first, and take $z_{4}$ moving along a closed orbit from the family $\left(p_{2}(t, h), q_{2}(t, h)\right)$ parametrized by the values $G=h$. Take $z_{3}$ moving along the separatrix $\left(\bar{p}_{1}(t), \bar{q}_{1}(t)\right)$ of $F$ given by

$$
t=4 \bar{p}_{1}+(8 / \sqrt{3}) \log \left[\left(\sqrt{3}-\bar{p}_{1}\right) /\left(\sqrt{3}+\bar{p}_{1}\right)\right] .
$$

As the parameter $\varepsilon$ is "turned on," these motions are perturbed. What happens can be physically described as follows:

Vortex $z_{4}$ motion is only slightly varied, but each time $z_{3}$ approaches one of the saddle points, it must "flip a coin" to choose which branch to follow (so escapes are also possible). Furthermore, if 5 vortices are considered (two strong and three weak) then Arnold diffusion occurs [HM1]. Here there is a slow transfer of amplitude between oscillations of two of the offsprings mediated by a third moving near the separatrix.

We observe that in order to apply [HM] it is not necessary to express the closed orbits $z_{4}$ in terms of action angle variables $I, \theta(I=I(h))$. Unfortunately, $z_{4}(t, h)$ cannot be given in terms of elementary functions, so the task of evaluating analytically the Melnikov function seems hopeless, at first sight. However, near $z_{1}=i$ we may write, up to $0\left(r^{2}\right)$ terms,

$$
p_{2}=r \sin \omega t, \quad q_{2}=1+r \cos \omega t, \quad \omega=1 / 2 r^{2} .
$$


It follows that the Melnikov formula of $[\mathrm{HM}]$ can now be explicitly evaluated by residues [KC1], yielding an asymptotic formula for $r \ll 1$. We do not present it here because it turns out that the result is the same for the unrestricted problem [KC2], which we will treat next.

\section{Melnikov's Integral for the Unrestricted Problem}

So far we have ignored the fact that the offsprings induce an $O(\varepsilon)$ perturbation on the couple. Physically, one may worry that the pair will eventually collide or split apart to infinity. Mathematically, horseshoes are features of Poincaré maps. Our construction depended on choosing a reference frame in uniform motion, and this is no longer possible in the general case.

In order to address these difficulties, we must consider the ODEs for the distances between the vortices. We recall from [A1] that for a system $z_{\alpha}, 1 \leqq \alpha \leqq m$, then $\ell_{\alpha \beta}=\left|z_{\alpha}-z_{\beta}\right|$ obey

$$
d / d t \ell_{\alpha \beta}^{2}=\sum^{\prime \prime} k_{\alpha} \sigma_{\alpha \beta \gamma} A_{\alpha \beta \gamma}\left(\ell_{\beta \gamma}^{-2}-\ell_{\gamma \alpha}^{-2}\right),
$$

where the sum extends over all $\gamma \neq \alpha, \beta ; A_{\alpha \beta \gamma}$ is the area of the triangle with vertices at $z_{\alpha}, z_{\beta}, z_{\gamma}$ (function of the distances via Perron's formula); $\sigma_{\alpha \beta \gamma}= \pm 1$ gives the orientation ( +1 for $z_{\alpha}, z_{\beta}, z_{\gamma}$ counterclockwise).

There are two integrals of motion,

$$
H=\sum_{1 \leqq \alpha<\beta \leqq m} k_{\alpha} k_{\beta} \log \ell_{\alpha \beta}, \quad G=\sum_{1 \leqq \alpha<\beta \leqq m} k_{\alpha} k_{\beta} \ell_{\alpha \beta}^{2} .
$$

Notice that there are only $2 n-3$ independent distances (say, given by $\ell_{12}$ and $\ell_{1 j}, \ell_{2 j}, j=3, \ldots, n$, forming $n-2$ triangles with a common side). Thus for $n=4$ there are 5 independent distances $\ell_{12}, \ell_{13}, \ell_{23}, \ell_{14}, \ell_{24}$ so (5) reduces immediately to an ODE on a five dimensional phase space. We want to use the integrals of motion (6) to further reduce the dynamics to a three dimensional phase space: we will then be in good shape to look for chaotic behavior.

Definition. The pseudo action-angle coordinates $(I, \theta)$ for $\ell_{14}, \ell_{24}$ are the action angle coordinates for $z_{4}$ motion on an "instantaneous" unperturbed restricted system (phase portrait given by Fig. 1) $z_{1}, z_{2}, z_{4}$.

This construction is reminiscent of the Keplerian "elements" in Celestial Mechanics. Notice that $\ell_{12}$ enters here as a parameter: $z_{4}=z_{4}\left(I, \theta ; \ell_{12}\right)$. Hence it follows that the dynamic variables of the problem can be replaced by

$$
I, \quad \theta, \quad \ell_{12}, \quad \ell_{13}, \ell_{23} \text {. }
$$

We introduce one more parameter $\varrho$ in the problem; namely, we do not assume that the weak vortices have equal strength: $k_{3}=\varepsilon \varrho, k_{4}=\varepsilon$. It will turn out that $\varrho$ does not appear in the final result.

Our strategy is to use the integrals of motion in order to eliminate the variables $I, \ell_{12}$. Then the three dimensional autonomous system for $\theta, \ell_{13}, \ell_{23}$, where the independent variable is the time, can be reduced to a two-dimensional nonautonomous system for $\ell_{13}, \ell_{23}$ (where the independent variable is $\theta$ ). The $O(\varepsilon)$ perturbation is $2 \pi$-periodic in $\theta$ and we can perform a Melnikov calculation. 
There is an immediate difficulty in this program, however: the integrals

$$
\begin{gathered}
H_{\varepsilon}=-k^{2} \log \ell_{12}+\varepsilon k\left(\varrho \log \ell_{13} / \ell_{23}+\log \ell_{14} / \ell_{24}\right)+\varepsilon^{2} \varrho \log \ell_{34}, \\
G_{\varepsilon}=-k^{2} \ell_{12}^{2}+\varepsilon k\left(\varrho \ell_{13}^{2}-\varrho \ell_{23}^{2}+\ell_{14}^{2}-\ell_{24}^{2}\right)+\varepsilon^{2} \varrho \ell_{34}
\end{gathered}
$$

are functionally dependent for $\varepsilon=0$. However, we may consider instead (this key idea was given to us by Prof. R. C. Robinson)

where

$$
\begin{gathered}
\widetilde{G}_{\varepsilon}=(1 / \varepsilon)\left[H_{\varepsilon}+k^{2} / 2 \log \left(-G_{\varepsilon} / k^{2}\right)\right], \quad \varepsilon \neq 0, \\
\tilde{G}_{0}=\lim _{\varepsilon \rightarrow 0} \tilde{G}_{\varepsilon}=k \varrho F\left(\ell_{13}, \ell_{23}\right)+k F\left(\ell_{14}, \ell_{24}\right),
\end{gathered}
$$

$$
F(a, b)=\log a / b-\left(a^{2}-b^{2}\right) / 2 \ell_{12}^{2} .
$$

It is interesting to observe that Hamiltonian (1) is recovered from (9) if we set $\ell_{12}=2\left(z_{1}=i, z_{2}=-i\right)$.

In Appendix A we outline the calculations leading to the following

Proposition 2. Using the integrals $H_{\varepsilon}=h, \widetilde{G}_{\varepsilon}=g$, one obtains the reduced system

$$
d / d \theta\left(\ell_{13}^{2}, \ell_{23}^{2}\right)=(1 / \omega(g, h))\left[\mathbf{f}\left(\ell_{13}^{2}, \ell_{23}^{2} ; h\right)+\varepsilon \mathbf{r}\left(\ell_{13}^{2}, \ell_{23}^{2}, \theta ; g, h, \varrho\right)\right]+O\left(\varepsilon^{2}\right),
$$

where $\omega(g, h)$ is the angular frequency for $\theta$ in the unperturbed motion of $z_{4}$, and

$$
\begin{gathered}
\mathbf{f}=k \sigma_{123} A_{123}\left(\ell_{23}^{-2}-\ell_{12}^{-2}, \ell_{13}^{-2}-\ell_{12}^{-2}\right), \\
\mathbf{r}=\mathbf{p}+\mathbf{q}, \\
\mathbf{p}=\left(\sigma_{134} A_{134}\left(\ell_{34}^{-2}-\ell_{14}^{-2}\right), \sigma_{234} A_{234}\left(\ell_{34}^{-2}-\ell_{24}^{-2}\right)\right), \\
\mathbf{q}=s \cdot \partial \mathbf{f} /\left.\partial \ell_{12}\right|_{\ell_{12}^{0}(h)}, \\
\ell_{12}^{0}=\exp \left(-h / k^{2}\right), \\
s=\left(\ell_{12}^{0} / k\right)\left(\varrho \log \ell_{13} / \ell_{23}+\log \ell_{14} / \ell_{24}\right) .
\end{gathered}
$$

Remark. The reader can verify easily that (9) is an integral for the planar vectorfield $\mathbf{f}$.

We need now a minor variant of Melnikov's formula in [HM], whose proof we give, for completeness, in Appendix B. Consider a system of ODE's

$$
\dot{\mathbf{x}}=\mathbf{f}(\mathbf{x})+\varepsilon \mathbf{r}(\mathbf{x}, t)+O\left(\varepsilon^{2}\right), \quad \mathbf{x}, \mathbf{f}, \mathbf{r} \in R^{2} .
$$

Assume that $\mathbf{r}$ is $T$-periodic in time, that the unperturbed system has a separatix curve $\bar{x}(t)$ and possesses moreover an integral of motion $F(x)$. Let $a \in(-\infty, \infty)$, and consider the normal line $L_{a}$ to the separatrix through $\bar{x}(a)$. It first meets the invariant curves $C_{\varepsilon}^{u}, C_{\varepsilon}^{s}$ of the Poincaré map (time $T$ return) on the points $x_{\varepsilon}^{u, s}(a)$. Using the level lines of $F$ to measure "distances" along $L_{a}$ one gets the Melnikov distance

$$
d(a, \varepsilon)=\left(\nabla F(\bar{x}(a)) \mid x_{\varepsilon}^{u}(a)-x_{\varepsilon}^{s}(a)\right)=\sum_{j=1}^{\infty} \varepsilon^{j} M^{(j)}(a) .
$$

Proposition 3 (Melnikov's criterion). (i) If $M=M^{(1)}$ changes sign at a, then for $\varepsilon$ small enough, $C_{\varepsilon}^{u}$ and $C_{\varepsilon}^{s}$ intersect transversally $\varepsilon$-near the point $\bar{x}(a)$ of the separatrix.

(ii) The first Melnikov function is given by

$$
M(a)=\int_{-\infty}^{\infty}(\nabla F(\bar{x}(t+a)) \mid \mathbf{r}(\bar{x}(t+a), t)) d t .
$$


Notice that the factor $\omega(g, h)$ in $(10 \mathrm{a})$ is immaterial for the sake of Melnikov's criterion. Moreover, the integral may be done directly with respect to time (instead of the angle $\theta)$, using the original distance variables.

The following result is derived in Appendix $C$ :

Proposition 4. The Melnikov function (13) for the data (10b) is written as

$$
M(a)=\int_{-\infty}^{\infty}\left(\ell_{13}^{-2}-\ell_{12}^{-2}\right)\left[\sigma_{134} A_{134}\left(\ell_{34}^{-2}-\ell_{14}^{-2}\right)-\sigma_{234} A_{234}\left(\ell_{34}^{-2}-\ell_{24}^{-2}\right)\right] d t,
$$

where the functions are evaluated at:

$$
\begin{gathered}
\ell_{12}^{0} \quad \text { (unperturbed distance between the primaries), } \\
\bar{\ell}_{13}(t+a / \omega), \bar{\ell}_{23}(t+a / \omega) \quad \text { (separatrix), } \\
\ell_{14}(t), \ell_{24}(t) \quad\left(\text { closed orbit of } z_{4}\right. \text { unperturbed motion), } \\
\ell_{34}=\ell_{34}\left(\ell_{12}^{0}, \bar{\ell}_{13}(t+a / \omega), \bar{\ell}_{23}(t+a / \omega), \ell_{14}(t), \ell_{24}(t)\right) .
\end{gathered}
$$

Notice that there is no dependence in the parameter $\varrho$. Symmetry considerations show that $M(a)$ is odd. If the zeros are simple, then it follows that the stable and unstable curves of the Poincare mapping from sections $\theta=0$ to $\theta=2 \pi$ intersect $\varepsilon$-near the points given by $\left(\bar{\ell}_{13}\left(t_{n}\right), \bar{\ell}_{23}\left(t_{n}\right)\right), t_{n}=n T / 2$, where $T$ is the period of the closed orbit described by $z_{4}$ in the unperturbed system. In order to show that the zeros are simple requires evaluating the Melnikov integral. Our final calculation is outlined in Appendix D. We summarize it as the following

Theorem. For $r \ll 1$ as in (4), the Melnikov function $M(a)=M(a, r)$ has a formal expansion

$$
M(a, r)=\sum_{n=1}^{\infty} r^{n} B_{n}(a, \omega), \quad \omega \sim 1 / 2 r^{2}
$$

where $B_{n}$ decreases exponentially in $\omega$ (the higher $n$ is the higher the rate). Moreover, the leading term is given by

$$
\pi r \exp \left[-\left(1 / 2 r^{2}\right)(8 \pi / 3 \sqrt{3}-4)\right] \cdot \sin (a) .
$$

Remark. The zeros $a_{n}=n \pi$ correspond to $t_{n}=2 r^{2} n \pi$.

\section{Discussion: Melnikov's Method vs. Lagrangian Intersection Theory}

We claim that non-integrability follows immediately from (15). However, we must address a serious point that was raised by the referee. We take the liberty to quote from his report:

\footnotetext{
"Apparently only the heteroclinic orbit connecting $\left(p_{1}, q_{1}\right)=( \pm \sqrt{3}, 0)$ is treated [Eq. (3)]. This is not enough to conclude that homoclinic cycles exist, since one must check that solutions can return from $(-\sqrt{3}, 0)$ to $(\sqrt{3}, 0)$ near the other unperturbed connections. [Fig. 1 is the unperturbed $\left(p_{1}, q_{1}\right)$ phase plane.] The authors must deal with this problem if they wish to use the "usual" proof of nonintegrability via construction of a "horseshoe-like" invariant Cantor set of recurrent points."
} 
In fact, the Dynamical Systems paradigm "transversal intersection of stable and unstable manifolds implies chaos" is not necessarily true for heteroclinic connections (see [Kz] or [D]). Thus, if the curved separatrices in Fig. 1 split open under the $O(\varepsilon)$ perturbation (in a Poincaré mapping of a suspension) then we could not claim that non-integrability follows from (15).

Although one expects, physically, that the curved separatrices will be also broken with transversal intersection, there are examples in which some separatrices are and others are not broken under Hamiltonian perturbations of integrable systems [Kz1]. However, as long as the curved separatrices do not split open (without intersection), the necessary recurrence needed to obtain a horseshoe map is guaranteed.

In fact, for Hamiltonian systems the perturbed stable and unstable manifolds must always intersect!

This follows from the Lagrangian intersection theory of Weinstein [W], as pointed out in [HM1, Sect. 2, Remark 4]. Moreover, transversal splitting is the generic situation.

What is, therefore, the point of taking the pains of evaluating Melnikov integrals? One answer would be that it allows one to analytically check transversality in specific cases, such as the one presented here. Another one, reversing the viewpoint, and perhaps more exciting, is that when the Melnikov function is identically zero (say, for particular choices of parameters) then one has a symptom of a possible integrability for the system. For instance, Kozlov has checked this for Kowalesvskaya's top [Kz].

A final remark is that, if one wants to avoid, at this stage, (i) invoking the highpowered Lagrangian intersection theory (perhaps an overkill) or (ii) performing a numerical evaluation of the Melnikov function along the curved separatrices (this would take away the punch, if any, of this paper), there would be still a way out. One could show, without any work, that all Melnikov integrals $M^{(j)}(a)$ vanish, at the parameter value $a$ corresponding to the points of the separatrices in the vertical axis of symmetry of Fig. 1. In fact, using a symmetry argument one can verify, by inspection, that the integrands are odd functions.

\section{Appendix A. Proof of Proposition 2}

We apply the implicit function theorem to $H_{\varepsilon}=h, \widetilde{G}_{\varepsilon}=g$, and solve for $\ell_{12}, I$. We need only

$$
\begin{gathered}
\ell_{12}=\ell_{12}^{0}+\varepsilon S, \quad \ell_{12}^{0}=\exp \left(-h / k^{2}\right), \\
s=\left(\ell_{12}^{0} / k\right)\left(\varrho \log \ell_{13} / \ell_{23}+\log \ell_{14} / \ell_{24}\right) .
\end{gathered}
$$

Consider Eqs. (5) for $\ell_{13}$ and $\ell_{23}$. They can be written as

$$
d / d t\left(\ell_{13}^{2}, \ell_{23}^{2}\right)=\mathbf{f}\left(\ell_{13}^{2}, \ell_{23}^{2} ; \ell_{12}^{2}\right)+\varepsilon \mathbf{p}\left(\ell_{13}^{2}, \ell_{23}^{2}, \ell_{14}^{2}, \ell_{14}^{2}, \ell_{34}^{2}\right),
$$

where $\mathbf{f}$ and $\mathbf{p}$ appear in (10b). The point to notice is that $\mathbf{r}$ carries a contribution $\mathbf{q}$ arising from $\mathbf{f}$, upon substituting (A.1). 


\section{Appendix B. Derivation of Melnikov's Formula (13)}

All Melnikov-type formulas are derived along the same lines. One combines variational equations with a tricky use of the Fundamental Theorem of Calculus. Write $x_{\varepsilon}^{s, u}(t, a)$, the solution of (11) with initial condition $x_{\varepsilon}^{s, u}(0, a)=x_{\varepsilon}^{s, u}(a) \in L_{a}$ $\cap C_{\varepsilon}^{s, u}$. Then

$$
x_{\varepsilon}^{s, u}(t, a)=\bar{x}(t+a)+\varepsilon \xi^{s, u}(t, a)+O\left(\varepsilon^{2}\right),
$$

where $\xi^{s, u}$ satisfies the variational equation (with different initial conditions)

$$
\dot{\xi}=D \mathbf{f}(\bar{x}(t+a)) \quad \cdot \xi+\mathbf{r}(\bar{x}(t+a), t)
$$

with $\xi^{s}\left(\xi^{u}\right)$ uniformly bounded for $t>0(t<0)$. Substituting (A.2) into (12) we get

$$
M(a)=\left(\nabla F(\bar{x}(a)) \mid \xi^{u}(a)-\xi^{s}(a)\right) .
$$

Now,

$$
\begin{aligned}
& \left(\nabla F\left(\bar{x}(a) \mid \xi^{u}(a)\right)=\int_{-T_{2}}^{0} d / d t\left(\nabla F \left(\bar{x}(t+a)\left|\xi^{u}(t, a) d t+\left(\nabla F \mid \xi^{u}\right)\right|_{t=-T_{2}},\right.\right.\right. \\
& \left(\nabla F\left(\bar{x}(a) \mid \xi^{s}(a)\right)=-\int_{0}^{T_{1}} d / d t\left(\nabla F\left(\bar{x}(t+a) \mid \xi^{s}(t, a)\right) d t+\left.\left(\nabla F \mid \xi^{s}\right)\right|_{t=T_{1}} .\right.\right.
\end{aligned}
$$

Letting $T_{1}, T_{2} \rightarrow \infty$, then $\nabla F\left(\bar{x}\left(T_{1}+a\right)\right), \nabla F\left(\bar{x}\left(-T_{2}+a\right)\right) \rightarrow 0$, and $\xi^{s, u}$ remain bounded, so the second terms above drop out. Moreover,

$$
\begin{gathered}
d / d t\left(\nabla F(\bar{x}(t+a) \mid \xi(t, a))=\left(d^{2} F \cdot \dot{\bar{x}} \mid \xi\right)+\left(\nabla_{\bar{x}} F \mid \dot{\xi}\right)\right. \\
=\left(d^{2} F \cdot \dot{\bar{x}} \mid \xi\right)+\left(\nabla_{\bar{x}} F \mid D f_{\bar{x}} \cdot \xi\right)+\left(\nabla_{\bar{x}} F \mid r\right),
\end{gathered}
$$

where we have omitted the superscripts ${ }^{s, u}$ and $\bar{x}(t+a)$ in the derivatives of $F$. The reader can check that the first two terms above cancel [since $F$ is an integral for $f$, then $(\nabla F \mid \mathbf{f})=0$; differentiate this identity and evaluate at $\xi]$. The third term yields (13).

\section{Appendix C. Proof of Proposition 4}

The integrand of the Melnikov function is written as

$$
(\nabla F \mid \mathbf{r})=(\nabla F \mid \mathbf{p})+\left(\nabla F \mid \partial \mathbf{f} / \partial \ell_{12}\right) \cdot s .
$$

We differentiate the identity $(\nabla F \mid \mathbf{f})=0$ with respect to the "parameter" $\ell_{12}$, obtaining

$$
\left(\nabla F \mid \partial \mathbf{f} / \partial \ell_{12}\right)=-\left(\nabla\left(\partial F / \partial \ell_{12}\right) \mid \mathbf{f}\right)
$$

Hence the Melnikov function is written as $M(a)=M_{1}(a)-M_{2}(a)$, where

$$
M_{1}(a)=\int_{-\infty}^{\infty}(F \mid \mathbf{p}) d t, \quad M_{2}(a)=\int_{-\infty}^{\infty}\left(\nabla\left(\partial F / \partial \ell_{12}\right) \mid \mathbf{f}\right) s d t
$$

We interpret the result as follows: the first term is calculated with $\ell_{12}^{0}$ and an unmodified perturbation $\mathbf{p}$. It corresponds to the Melnikov function for the 
restricted problem and yields the expression in Proposition 4. The second term carries the parameter $\varrho$ (which appears in $s$ ), but

$$
\left(\nabla\left(\partial F / \partial \ell_{12}\right) \mid f\right)=k \ell_{12}^{-3} \sigma_{123} A_{123}\left(\ell_{23}^{-2}-\ell_{13}^{-2}\right)
$$

vanishes identically, since $\ell_{23}=\ell_{13}$.

\section{Appendix D. Setting Up the Residue Calculation}

Using (4), we have

$$
\begin{aligned}
& \ell_{12}^{2}=4, \quad \ell_{14}^{2}=r^{2}, \quad \ell_{13}^{2}=1+u^{2}, \\
& \ell_{34}^{2}=(u-r \sin \theta)^{2}+(1+r \cos \theta)^{2}, \quad \ell_{24}^{2}=r^{2} \sin ^{2} \theta+(2+r \cos \theta)^{2}, \\
& 2 \sigma_{134} A_{134}=\left|\begin{array}{ccc}
0 & 1 & 1 \\
u & 0 & 1 \\
r \sin \theta & 1+r \cos \theta & 1
\end{array}\right|=r(\sin \theta+u \cos \theta) \\
& 2 \sigma_{234} A_{234}=\left|\begin{array}{ccc}
0 & -1 & 1 \\
u & 0 & 1 \\
r \sin \theta & 1+r \cos \theta & 1
\end{array}\right|=-r \sin \theta+u(2+r \cos \theta) \text {. }
\end{aligned}
$$

Here $u=\bar{p}_{1}$ ranges in the interval $(-\sqrt{3}, \sqrt{3})$ via (3). Inserting into the integrand of the Melnikov function (Proposition 4), and changing the variable of integration $t$ to $u$, we get, after some manipulations,

$$
\begin{aligned}
& M(a, r)=-\frac{1}{2} \int_{-\sqrt{3}}^{\sqrt{3}} d u\left\{r(\sin \theta+u \cos \theta)\left[\frac{1}{\left(u-r \sin ^{2} \theta\right)^{2}+(1+r \cos \theta)^{2}}-\frac{1}{r^{2}}\right]\right. \\
& \left.-[u(2+r \cos \theta)-r \sin \theta]\left[\frac{1}{(u-r \sin \theta)^{2}+(1+r \cos \theta)^{2}}-\frac{1}{4+r^{2}+4 r \cos \theta}\right]\right\} .
\end{aligned}
$$

At first glance, there is a term diverging as $1 / r$, but its coefficient vanishes when evaluated by residues. The strategy for the calculation goes as follows: we treat $\omega$ in (4) as if it were independent of $r$, so formally

$$
M(a, r)=\sum_{n=1}^{\infty} r^{n} B_{n}(a, \omega) .
$$

We expanded (A.5) in powers of $r$, and performed the residue integrals [KC2]. The calculations are "tedious but straightforward." We observed that the $B_{n}$ decrease exponentially with respect to $\omega$ as $\omega \rightarrow \infty$. Moreover, the higher $n$, the higher the rate. The leading term is (15).

Acknowledgements. We thank Prof. J. E. Marsden for introducing us to the world of vortices, Prof. H. Aref for a guided tour on it, and Prof. R. C. Robinson for a key suggestion. One of us (JK) wishes to thank Prof. João Candido Portinari for forcing him to learn residue calculus as an undergraduate. We thank IMPA and $\mathrm{LNCC} / \mathrm{CNPq}$ for printing the technical reports $[\mathrm{KC1}$, KC2]. Last, but not least, JK wishes to thank Prof. V. Moncrief for sponsoring a visit to Yale University. 


\section{References}

[A1] Aref, H.: Integrable, chaotic, and turbulent vortex motion in two-dimensional flows. Ann. Rev. Fluid Mech. 15, 345-389 (1983)

[A2] Aref, H.: Chaos in the dynamics of few vortices. In: Theoretical and applied mechanics. Niordson, F., Olhoff, N. (eds.), pp. 43-68. Amsterdam: North-Holland 1985

[A3] Aref, H.: The numerical experiment in fluid mechanics. J. Fluid Mech. 173, 15-41 (1986)

[CB] Couder, Y., Basdevant, C.: Experimental and Numerical study of vortex couples in twodimensional flows. J. Fluid Mech. 173, 225-251 (1986)

[D] Devaney, R.L.: Transversal homoclinic orbits in an integrable system. Am. J. Math. 100, 631-642 (1978)

[GH] Guckenheimer, J., Holmes, P.J.: Nonlinear oscillations, dynamical systems and bifurcations of vectorfields. Berlin, Heidelberg, New York: Springer 1983

[HM] Holmes, P.J., Marsden, J.E.: Horseshoes in perturbations of Hamiltonian systems with two degrees of freedom. Commun. Math. Phys. 82, 523-544 (1982)

[HM1] Holmes, P.J., Marsden, J.E.: Melnikov's method and Arnold diffusion for perturbations of integrable Hamiltonian systems. J. Math. Phys. 23, 669-675 (1982)

[KC1] Koiller, J., Carvalho, S.: Non-integrability of a restricted problem of four point vortices. IMPA/Brazil A-022/83, Rio de Janeiro (1983)

[KC2] Koiller, J., Carvalho, S.: Chaos and non-integrability of point vortex motions. Report 010/85, LNCC/CNPq, Rio de Janeiro (1985)

[Kh] Khanin, K.M.: Quasi-periodic motions of vortex systems. Physica 4D, 261-269 (1982)

[Kz] Kozlov, V.V.: Integrability and non-integrability in Hamiltonian mechanics. Russ. Math. Surv. 38, 1-76 (1983)

[Kz1] Kozlov, V.V.: Phenomena of non-integrability in Hamiltonian systems. Proc. Intl. Congress. Math. 1986 (to appear)

[O] Ottino, J.M. et al: Morphological structures produced by mixing in chaotic flows. Nature 333, 419-425 (1988)

[W] Weinstein, A.: Lagrangian submanifolds and hamiltonian systems. Ann. Math. 98, 377-410 (1973)

[WS] Whipple, A.L., Szebehely, V.: The restricted problem of $n+v$ bodies. Cel. Mech. 32, 137-144 (1984)

[Z1] Ziglin, S.L.: Non-integrability of a problem on the motion of four point vortices. Sov. Math. Dokl. 21, 296-299 (1980)

[Z2] Ziglin, S.L.: Concerning the paper "Horseshoes in perturbations of Hamiltonian systems with two degrees of freedom" by P.J. Holmes and J.E. Marsden (Comments). Commun. Math. Phys. 108, 527-528 (1987)

Communicated by J. N. Mather

Received May 4, 1987; in revised form August 19, 1987 